\title{
Home Monitoring in Glaucoma
}

\author{
Toby Boote ${ }^{1}$ and Andrew J Tatham ${ }^{1,2}$ \\ 1. University of Edinburgh, Edinburgh, UK; 2. Princess Alexandra Eye Pavilion, Edinburgh, UK
}

DOl: https://doi.org/10.17925/EOR.2018.12.2.102

A wide range of relatively cheap, wearable digital devices are now available that enable self-monitoring of parameters such as heart rate, blood pressure, physical activity and sleep patterns. Although these devices are often marketed at healthy people, new technologies that permit home monitoring may also have the potential to improve the detection and management of chronic diseases including glaucoma. If patients were able to measure their intraocular pressure or perform perimetry at home, many more measurements could be obtained, with the potential to improve our ability to differentiate true change from test-retest variability. Home monitoring may also improve our ability to determine efficacy of treatment and enhance patient engagement.

\section{Keywords}

Glaucoma, home monitoring, intraocular pressure, IOP, visual field, Icare HOME, perimetry, tonometry

Disclosures: Toby Boote and Andrew J Tatham have nothing to declare in relation to this article.

Review Process: Double-blind peer review.

Compliance with Ethics: This study involves a review of the literature and did not involve any studies with human or animal subjects performed by any of the authors.

Authorship: The named authors meet the International Committee of Medical Journal Editors (ICMJE) criteria for authorship of this manuscript, take responsibility for the integrity of the work as a whole, and have given final approval to the version to be published.

open Access: This article is published under the Creative Commons Attribution Noncommercial License, which permits any noncommercial use, distribution, adaptation, and reproduction provided the original author(s) and source are given appropriate credit. (C) The Authors 2018.

Received: 4 September 2018

Accepted: 28 November 2018

Citation: European Ophthalmic Review. 2018;12(2):102-4

Corresponding Author: Andrew J Tatham, Princess Alexandra Eye Pavilion, 45 Chalmers Street, Edinburgh, EH3 9HA, UK. E: andrewjtatham@gmail.com

Support: No funding was received for the publication of this article.
The number of people living with chronic conditions is rapidly growing, increasing pressure on healthcare providers. In England, for example, it is estimated that long-term conditions account for $70 \%$ of total health and social care spending. 'Given that the average age of European populations is increasing and that for many diseases treatment costs are escalating, there is growing strain on the ability to deliver sustainable healthcare services.

Innovations in service delivery may help overcome some of the increased demand in managing chronic disease; for example, virtual clinics are now widely used for review of patients with glaucoma. In virtual clinics, patient data are collected by technicians or non-specialist optometrists, with an ophthalmologist reviewing the results remotely to decide whether the patient is stable or a change in treatment is required. A recent survey of UK ophthalmologists reported $50 \%$ of responders were based at a unit where glaucoma virtual clinics were already being used and a further $21 \%$ were planning to establish one. ${ }^{2}$ Virtual clinics are often run in hospital outpatient departments but can be based in mobile units or in any facility with space for the testing equipment, potentially reducing travel time for patients and allowing clinics to be held in communities without easy access to eye care services.

The management of chronic diseases may also be improved by the use of new technologies and it has been proposed that simple digital technologies such as smartphone applications could be applied to a healthcare setting to better inform patients to make healthier choices, improve access to services and provide information that allows healthcare professionals to deal more effectively with illness when it arises. ${ }^{3}$ New technology may also make it possible for patients to monitor health or disease in the comfort of their own home.

Home or self-monitoring of chronic diseases is certainly not a new concept and for diseases such as type 1 diabetes it is clearly essential. Telehealth is also becoming more frequently used in the management of systemic hypertension, where home monitoring helps to provide greater insight into blood pressure fluctuations. ${ }^{4}$ Self-monitoring is also increasingly used by healthy people as a wide range of relatively cheap, wearable digital devices are now available that enable measurements of parameters such as physical activity, heart rate, blood pressure and sleep patterns. Self-monitoring has entered the mainstream, but is it relevant to glaucoma?

\section{Home visual field monitoring}

Optimal detection and monitoring of glaucoma require measurement of intraocular pressure (IOP) and assessment of functional and structural parameters. All measurements are subject to test-retest variability and sometimes it is difficult to be certain whether changes are genuine or due to noise, though confidence can be improved by increasing the number of tests. For example, Chauhan et al. calculated that even if there was low test-retest variability it would take 6 years to detect a relatively fast progression rate of $1 \mathrm{~dB}$ per year with $80 \%$ power if only one visual field test was performed per year, but this could be reduced to 2 years if three examinations were performed each year. ${ }^{5}$ The recommendation was that six visual field tests be performed in the first 2 years after diagnosis. In clinical practice the frequency of visual field testing may be substantially less than these recommendations, which may contribute to the number of patients 
becoming visually impaired while under care. Home monitoring of visual fields may provide a means to increase the frequency of testing without increasing the number of clinic visits. Low-cost home visual field testing may also improve the detection of glaucoma and reduce the number of patients with advanced visual field loss at the time of diagnosis. Several investigators are developing vision-testing software for smartphones, tablet computers or laptops, or small, portable, purpose-built perimetry devices, which may have potential for home testing.

The Moorfields Motion Displacement Test (MMDT; London, UK) is a perimetric test that can be used on a standard laptop computer. In clinical tests the MMDT has been shown to have good ability to detect glaucoma with a sensitivity of $88.5 \%$ at a specificity of $85.0 \% .{ }^{6}$ The Melbourne Rapid Fields iPad Perimetry application (Glance Optical Pty Ltd., Syndal, Victoria, Australia) is an alternative test that can be performed rapidly on a standard iPad. The Melbourne application has comparable performance to standard automated perimetry for detection of glaucoma, with good test-retest reliability. Another free iPad application is the visualFields easy application (George Kong Software, Syndal, Victoria, Australia), which similarly has been shown to be a fast, effective and accurate method of visual field assessment. ${ }^{8}$ Moreover, a free internet-based visual field test, the Damato Multifixation Capimetry Online, shows promise to identify glaucomatous visual field defects. ${ }^{9}$

Anderson et al. evaluated the potential benefit of the increased frequency of testing made possible by home visual field monitoring compared to standard annual and biannual testing in the clinic. ${ }^{10}$ By simulating a variety of scenarios involving various test frequencies and test reliability they found home testing with the Melbourne Rapid Fields App (at monthly, two-weekly or weekly intervals) could improve ability to detect visual field progression. To achieve $80 \%$ sensitivity for detecting a $2 \mathrm{~dB}$ per year rate of visual field loss, biannual testing in the clinic would require 2.5 years of follow up, reducing to only 0.9 years with weekly home monitoring, even if the patient was only moderately compliant with instructions for weekly testing (63\%). Home monitoring of visual field therefore shows great promise and may also lead to diagnostic tests being affordable and available to individuals with limited access to healthcare.

\section{Home intraocular pressure monitoring}

IOP measurements also fluctuate; however, fluctuation is due to diurnal variation as well as measurement variability. Studies from sleep laboratories have shown up to $75 \%$ of individuals have peak IOP outside office hours, with the result that it may be difficult to determine true peak IOP and the possible effect of IOP fluctuation on progression. ${ }^{11}$ Having access to only a limited number of IOP measurements may also make it difficult to differentiate treatment effect from an apparent reduction in IOP due to regression to the mean. A fuller understanding of IOP fluctuation can be obtained by admitting patients for repeat measurements; however, this is costly and logistically difficult. Recently, a novel device designed for home IOP monitoring has become available, the Icare ${ }^{\circledast}$ HOME (TA022; Icare Finland Oy, Vanda, Finland) (Figure 1).

The Icare HOME is a rebound tonometer that quantifies IOP by measuring the deceleration of a magnetised disposable probe as it rebounds from the surface of the cornea. The device does not require instillation of topical anaesthesia and has been reported to provide repeatable readings with good agreement with Goldmann applanation tonometry. ${ }^{12-14}$ The Icare HOME can be linked to the patient's smartphone and IOP measurements uploaded to the cloud, allowing the clinician remote access to data.

\section{Figure 1: The Icare ${ }^{\circledast}$ HOME rebound tonometer}

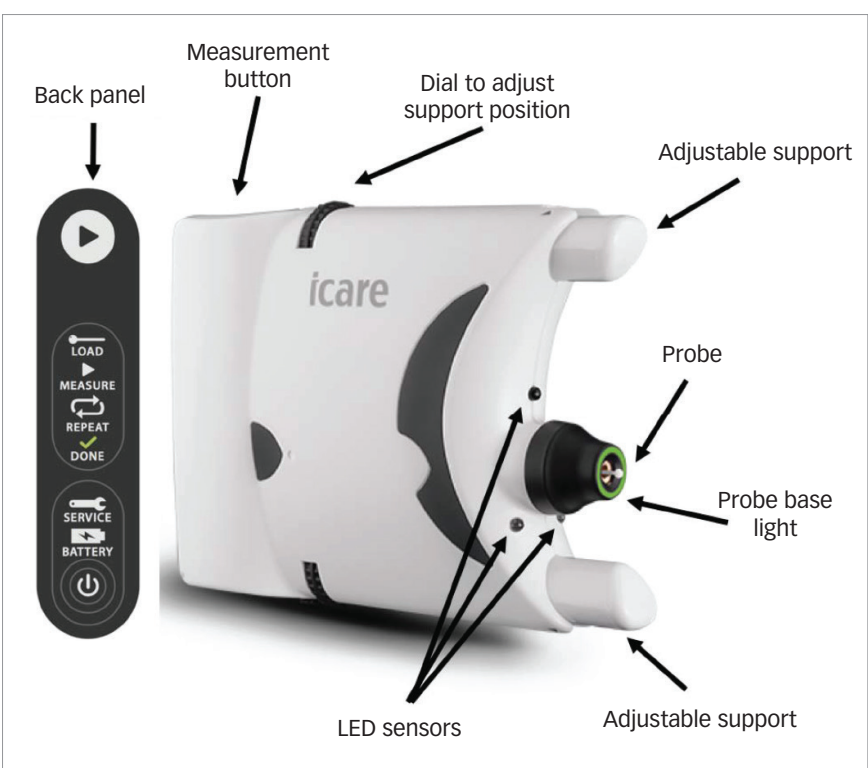

LED = light-emitting diode.

Icare HOME (TA022; ICare Finland Oy, Vanda, Finland.)

We recently conducted a study to examine whether patients were able to measure their own IOP and found $73 \%$ of patients could perform self-tonometry and obtain IOP measurements within $5 \mathrm{mmHg}$ of those obtained by a clinician. ${ }^{14}$ IOP measurements from Icare HOME were on average $2.66 \mathrm{mmHg}$ lower than those from Goldmann applanation tonometry; however, the Icare HOME had good reproducibility (intraclass correlation coefficient $=0.903 ; 95 \%$ confidence interval: 0.867-0.928). We also surveyed patients' perceptions of home tonometry and found more than $90 \%$ of those able to measure their own IOP were interested in using self-tonometry in the future, ${ }^{14}$ suggesting there is great interest among patients in supplementing clinic visits with home disease monitoring.

New technologies such as contact lens sensors could also provide a means for home IOP monitoring and there is some evidence that measurements from these sensors could be useful for predicting risk of glaucoma progression. ${ }^{15}$ Contact lens sensors also have the advantage of providing almost continuous measurements; however, they obscure vision, can only be used for a maximum of 24 hours and IOP patterns may be different on different days.

\section{Potential challenges}

To be a sound telemedical technology, it is essential that data collected from home monitoring devices are appropriately stored (e.g. using encrypted data connections) and analysed. ${ }^{16}$ One of the challenges of home IOP monitoring is that the quantity of data obtained is vastly greater than that obtained through office-hour IOP measurement. The quantity of data may be overwhelming for the patient and clinician and without guidance the patient may become anxious due to isolated high IOP measurements. Further, due to the paucity of IOP measurements traditionally obtained, there is still poor understanding of which 24-hour IOP parameter is most significant and whether knowledge of IOP fluctuations adds value to glaucoma management.

It is also important to appreciate that although patients seem interested in home monitoring for glaucoma, it is conceivable that enthusiasm may diminish with long-term use. This has been observed 
in studies of home blood-pressure monitoring where longer studies tend to have higher rates of poor adherence to self-monitoring compared with studies of shorter duration. ${ }^{4}$ The cost-effectiveness of home monitoring devices for glaucoma also still needs to be proven. Moreover, Mudie et al. evaluated Icare HOME and found that from 189 recruited patients, $4 \%$ failed to complete the study due to time concerns and $6 \%$ had difficulty using the device. ${ }^{17}$ Further studies are required to assess the validity of home tonometry.

\section{Continuous intraocular pressure monitoring strategies}

In theory, the ideal IOP monitoring device would be able to take measurements continuously to enable a better understanding of a patient's fluctuations and the impact that has on their glaucoma. Factors such as the time of day, stress, body posture and blood pressure can impact IOP. 18,19 Furthermore, accuracy of measurements can be improved by using implantable devices - such devices can measure IOP directly rather than perceiving it from the pressure of the cornea or sclera.

A study described how wearable, smart contact lenses can monitor both blood glucose levels and IOP simultaneously on in vivo and in vitro models. ${ }^{20}$ The data is then wirelessly and continuously sent to a device for analysis and monitoring. Additionally, IOP measurements obtained from a continuous contact lens sensor were found to be accurate and may be useful at detecting patients at higher risk of glaucoma progression. ${ }^{15}$ As yet, there is insufficient data to assess the suitability of these smart contact lenses as telemedical devices, but they would likely cost less to produce and so may be more cost effective.
Lee et al. reported the development of a nanoengineering device capable of continuously monitoring IOP for up to 4.5 months in in vivo rabbit models. ${ }^{21}$ Sensors were secured inside the anterior chamber of rabbit eyes and were found to be accurate to within $0.29 \mathrm{mmHg}{ }^{21}$ This device has the potential to enable convenient, comfortable, accurate and on-demand home monitoring of a patient's IOP, requiring no skill or patient compliance for use. More research is required to assess its use in patients with glaucoma and to ascertain if there are any complications from such a device.

Overall, wearable IOP sensors tend to be safer and easier to design but they may cause tissue changes. ${ }^{22}$ Implantable sensors are more accurate than wearable IOP sensors but require a surgical procedure and have higher clinical restrictions such as the requirement to ensure accurate IOP measurement for a longer period with minimal discomfort to the patient. ${ }^{23}$

\section{Conclusion}

The management of glaucoma requires frequent monitoring and while measurements of parameters such as IOP and visual field are traditionally only obtained by visits to healthcare professionals, devices are now available that allow patients to obtain some of these measurements through self-testing. Home monitoring has the advantage of providing a greater quantity of data, which may improve understanding of treatment efficacy and help us better differentiate true change from measurement variability. Home monitoring may also improve patient engagement; however, further evidence is needed to determine whether it is cost effective and whether access to greater quantity of data improves outcomes for patients. $\square$
1. Department of Health. Improving the health and well-being of people with long term conditions. London: Department of Health, 2013. Available at: www.yearofcare.co.uk/sites/default/ files/pdfs/dh_improving\%20the\%20h\&wb\%20of\%20people\%20 with\%20LTCs.pdf (accessed 19 December 2018)

2. Gunn PJG, Marks JR, Au L, et al. Acceptability and use of glaucoma virtual clinics in the UK: A national survey of clinical leads. BMJ Open Ophthalmol. 2018;3:e000127.

3. Department of Health. Personalised health and care 2020: framework for action. London: Department of Health, 2014. Available at: www.gov.uk/government/publications/personalisedAvailable at: www.gov.uk/government/publications/personalise-
health-and-care-2020/using-data-and-technology-to-transformoutcomes-for-patients-and-citizens (accessed 19 December 2018.

4. Harrison C, Wild K. Using telehealth in the management of hypertension. Nurs Stand. 2017;31:44-9.

5. Chauhan BC, Garway-Heath DF, Goñi FJ, et al. Practical recommendations for measuring rates of visual field change in glaucoma. Br J Ophthalmol. 2008;92:569-73.

6. Ong EL, Zheng Y, Aung T, et al. Performance of the Moorfields motion displacement test for identifying eyes with glaucoma. Ophthalmology. 2014;121:88-92.

7. Prea S, Kong Y, Mehta A, et al. Six-month Iongitudina comparison of a portable tablet perimeter with the Humphrey Field Analyzer. Am J Ophthalmol. 2018:190:9-16.
8. Johnson C, Thapa S, George Kong Y, Robin A. Performance of an iPad application to detect moderate and advanced visual field loss in Nepal. Am J Ophthalmol. 2017;182:147-54.

9. Olsen A, Alberti M, Serup L, et al. Glaucoma detection with damato multifixation campimetry online Eye. 2016:30:731-39.

10. Anderson A, Bedggood P, George Kong Y, et al. Can home monitoring allow earlier detection of rapid visual field monitoring allow earlion in glaucoma? Ophthalmogy 2017:124:1735-42.

11. Liu JH, Kripke DF, Twa MD, et al. Twenty-four-hour pattern of intraocular pressure in the aging population. Invest Ophthalmol vis Sci. 1999:40:2912-17.

12. Noguchi A, Nakakura S, Fujio Y, et al. A pilot evaluation assessing the ease of use and accuracy of the new self/hometonometer Icare HOME in healthy young subjects. J Glaucoma. 2016;25:835-41.

13. Dabasia PL, Lawrenson JG, Murdoch IE. Evaluation of a new rebound tonometer for self-measurement of intraocular pressure. Br J Ophthalmol. 2016;100:1139-43.

14. Pronin S, Brown L, Megaw R, Tatham A. Measurement of intraocular pressure by patients with glaucoma. JAMA Ophthalmol. 2017:135:1030.

15. De Moraes C, Jasien J, Simon-Zoula S, et al. Visual field change and 24-hour IOP-related profile with a contact lens sensor in treated glaucoma patients. Ophthalmology 2016;123:744-53

16. Klonoff D. Diabetes and telemedicine: Is the technology sound, effective, cost-effective, and practical? Diabetes Care. 2003;26:1626-8.

17. Mudie L, LaBarre $S$, Varadaraj V, et al. The Icare HOME (TA022) Study. Ophthalmology. 2016;123:1675-84.

18. Jindal V. Glaucoma: A multifactorial disease and its multidimensional management. Int I Sci Res. 2013:3:1-3.

19. Whitacre MM, Stein R. Sources of error with use of Goldmann type tonometers. Surv Ophthalmol. 1993;38:1-30.

20. Kim J, Kim M, Lee M, et al. Wearable smart sensor systems integrated on soft contact lenses for wireless ocula diagnostics. Nat Commun. 2017;8:14997.

21. Lee J, Park H, Du J, et al. A microscale optical implant for continuous in vivo monitoring of intraocular pressure. Microsyst Nanoeng. 2017;3:17057.

22. Molaei A, Karamzadeh V, Safi S, et al. Upcoming methods and specifications of continuous intraocular pressure. monitoring systems for glaucoma. J Ophthalmic Vis Res. 2018;13:66.

23. Clausen I, Glott T. Development of clinically relevant implantable pressure sensors: Perspectives and challenges. Sensors (Basel). 2014:14:17686-702 